Rev. Elev. Méd. Vét. Pays trop., 1967, 20, 3 (457-473)

\title{
Premières remarques écologiques concernant quelques espèces d'Oribates au Tchad
}

\author{
J. GRUVEL ef $M$. GRABER \\ (Note préliminaire)
}

\begin{abstract}
RÉSUMÉ
Les nombreuses récoites d'Acariens Oribates effectuées au Tchad depuis 1963 dans le but d'étudier la transmission du Cestode du mouton Stilesia globipunctato ont permıs de rassembler quelques observations relatives à leur écologie.

Vingt-trois espèces d'Oribates ont été récoltées en différents points des terrains du Laboratoire de Farcha, sıtué en zone sahélienne et dont les caractéristiques cl imatiques et botaniques sont brıèvement rappelées.

Les Oribates sont toujours obtenus en abondance tout au long de l'année, mais Ils sont particulièrement nombreux en saison fraîche.

Parrni les espèces dénombrées, quelques-unes seulement sont constamment rencontrées quels que soient la saıson et le lıeu ; toutes les auires élant très fajblement représentées, voire même exceptionnelles.

Après la saison des pluies, des études systématıques ont été réalisées dans un gisement particulièrement riche. A chaque heure de la journée et à trois niveaux différents ont été effectués des prélèvements. L'interprétation des résultats montre quelques aspects du comportement des espèces les plus abondantes.

Un rapport étroit entre les densités ef les mouvements des populations d'Oribates et les intensités d'infestations des moulons a cinsı été mis en évidence.
\end{abstract}

Depuis 1963, les sections de Parasitologie du Laboratoire de Farcha (Tchad) onf entrepris une étude sur les modes de transmission de certains Cestodes appartenant à la famille des Thysonosominae.

Cette étude, suscitée par l'importance des Cestodoses des ruminants dans ce territoire d'Afrique Centrale, a conduit à développer des recherches relatives aux Acariens Oribates, habituellement reconnus comme hôtes intermédiaires dans le développement de ces parasites. II a été aınsı démontré que Stilesia globipunctato Rivolta 1874, Cestode le plus répandu chez les ovins, pouvait être transmis par sIx espèces d'Orıbates les plus fréquemment rencontrées :

Scheloribotes perforofus Wellwork 1.964 ;

Scheloribates parvus van Pletzen conglobatus Wall. 1964 ;

Scheloribates fimbriatus Thor africonus Wallwork 1964:

Africacarus calcoratus Wallwork 1965 ;

Galumna bologhi Wallwork 1965 ;

Allogalumna pellucido Wallwork 1965.

La présente note a pour but de mettre en évidence quelques aspects de l'écologie des Oribates des environs de Fort-Lamy. 


\section{ÉTUdES GÉNÉRALES DU MILIEU}

\section{Situation.}

Les recherches d'Oribates n'ont jusqu'à présent été effectuées que dans les terraıns du Laboratoire de Farcha, situé en bordure du Chari, à quelques kilomètres au nord de Fort-Lamy. Répondant à peu près aux coordonnées géographiques, $15^{\circ}$ de longitude Est et $12^{\circ} 10^{\prime}$ de latitude Nord: les points de récolte se situent donc dans la partie nord de la zone climatique de type sahélo-soudanıen telle que la définit AUBREVILLE, en un lieu où la moyenne annuelle des précipitations est de $500 \mathrm{~mm}$. Celles-ci s'effectuent pendant trois mois, de juillet à octobre. Deux périodes sèches, fraîche de décembre à février, puis chaude de mars à juin, occupent le reste de l'année.

Les températures moyennes annuelles sont de $20,2^{\circ} \mathrm{C}$, pour les minimums et $35,6^{\circ} \mathrm{C}$ pour les maximums; l'humidité relative moyenne annuelle minimum étant de .18 p. 100 et maximum de 98 p. 100. Les variations de température et d'humidité sont ainsi très marquées au cours d'une année. Le graphique $n^{0} 1$ indique les valeurs moyennes mensuelles de ces deux éléments climatıques pour l'année 1964.
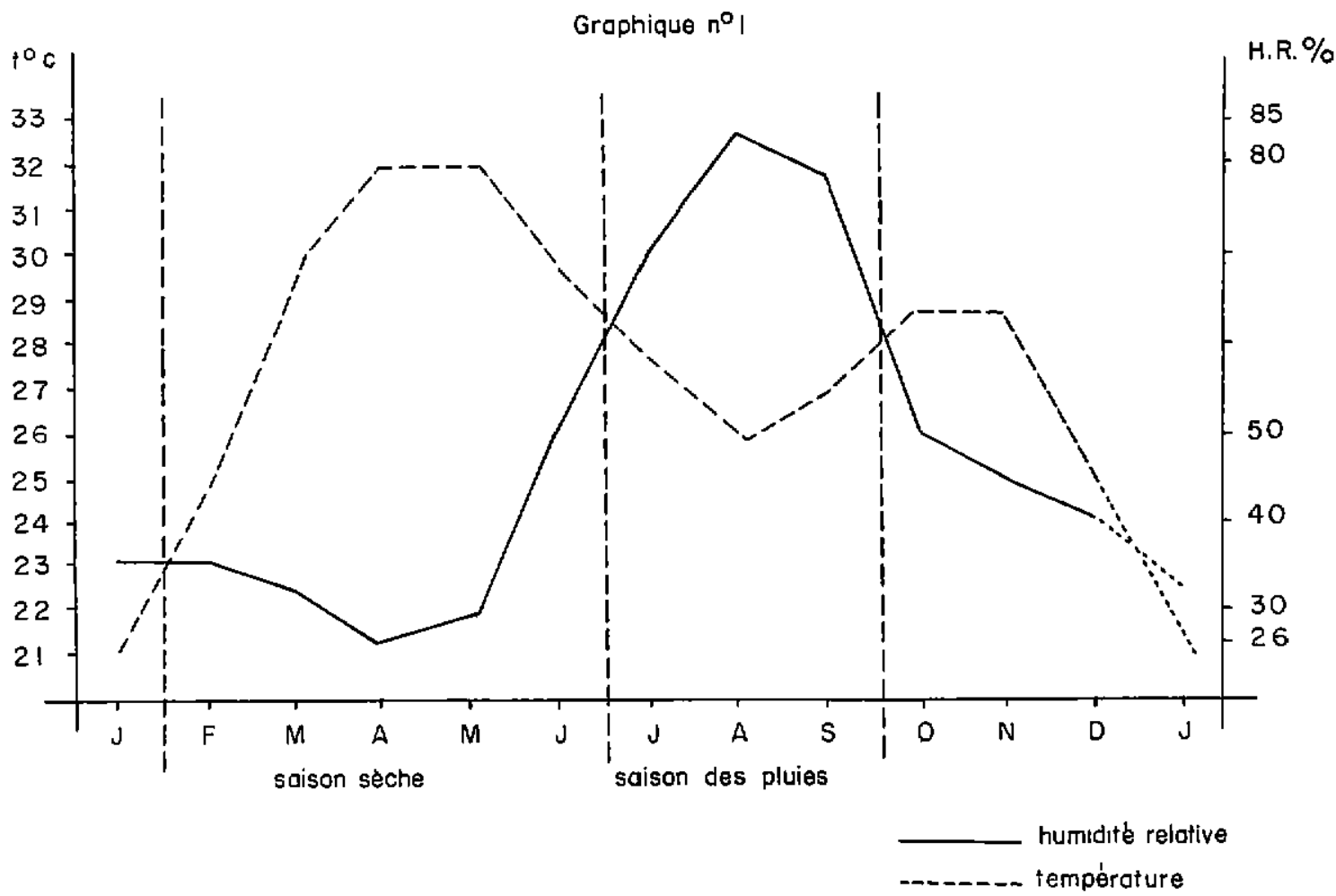

\section{Végétation $\left(^{*}\right)$.}

La végétation de la régıon de fort-Lamy est celle d'une savane arbustive claire, avec quelques arbres de pius de sept mètres de hauteur.

La végétation des stations du Laboratoire correspond à ce type, mais modifiée par l'ımplantation humaine. Quatre espèces : Tamorindus

* La rédaction de ce paragraphe a été effectuée sous les conseils de M. A. GASTON, Agrostologue au Laboratoire, qui doit être vivement remercié. indica L. (Caesalpmiacaea), Acacia sieberiona D. C. (Mimosacaea), Bolanites aegyptiaca L. Del (Zygophyllocaea) et Diospyros mespiliformis Hochst. ex D (Ebenacaea) ont persisté et constituent avec Azadirachto indica A. Juss. (Méliacaea) et Parkinsonia aculeota L. (Caesalpinlacoca), introduits récemment, la strate arborée des principaux gisements étudiés.

Ont persisté également Cadabo farınosa Forsk. (Capparidacaea), Capparis corymbosa Lam. (Capporidocaeo) et Boscia senegalensis (Pers.) Lam. 
ex Pois. qui ne se retrouvent qu'en sous-étage, sous forme d'arbrisseaux rabougris de $50 \mathrm{~cm}$ à $1 \mathrm{~m}$ de haut. La strate inférleure est presque exclusivement constituée d'herbes annuelles qui accomplissent leur cycle pendant la saison des pluies où elles donnent un recouvrement de 100 p. 100 . Elles sèchent dès le mois d'octobre et finissent par constıtuer sur le sol une litière de paille brisée.

La végétation des gisements se présente en définitive sous l'aspect d'un arbre isolé recouvrant de rares arbrisseaux et la strate annuelle.

\section{ÉTUDE GLOBALE DES ORIBATES}

\section{Prélèvements.}

La méthode de prélèvements par lavage est désarmais la seule utilisée pour les récoltes d'Oribates; son principe $a$ déjà été précisé dans une étude antérieure. Cette technique d'extraction présente l'ınconvénient de détruire les stades immatures dont l'ımportance permet d'apprécier la vitalité d'une population d'Oribates; mais elle a toujours aboutit à l'isolement de très nombreux Acariens, indispensables pour les études d'infestations expérimentales. C'est pour cette raison que, malgré ses inconvénients, cette méthode est encore retenue.

\section{$2 \circ$ Echantillons.}

Les échantillons, d'un volume pratıquement constant, d'environ $500 \mathrm{~cm}^{3}$, sont prélevés le plus souvent chaque matin dans les 12 différents gisements prospectés, à des profondeurs varıables mais n'excédant pas $40 \mathrm{~cm}$.

\section{$3^{\circ}$ Espèces récoltées.}

Ainsi, depuls 1963, 23 espèces d'Oribates onf été récoltées dans les terrains du Laboratoire. Parmi elles, on remarque 18 espèces et sousespèces nouvelles. Les déterminations dues à l'obligeance de Monsieur WALL-WORK de Londres, permettent d'en dresser la liste sulvante :

a) Famille Epilohmannudae Oudms 1923. Epilohmonnia cylindrico Berl.

b) Famılles Lohmanniidoe Berl. 1916. Heptacarus hirsufus n. sp. Wallwork 1964.

c) Famılle Oppiidae Grandj. 1954. Oppio heterosa n. sp. Wallwork 1964.
Oppio pilosello Bal. ssp. longiseta n. ssp. Wallwork 1964.

Oppia fusiformis n. comb. Wallwork 1964. Oppia fusiformis forma lyroseta $n$. form. Wallwork 1964.

d) Famille Passalozetidae Grandj. 1954.

Possalozetes pectinatus n. sp. Wallwork 1964.

e) Famille Oribatulidae Jacot 1929.

Oribatula acuminato n. sp. Wallwork 1964. Zygoribatula setosa Evans.

f) Famille Scheloribotidae Grandj. 1933.

Scheloribates perforatus n. sp. Wallwork 1964.

Scheloribotes fimbriatus Thor ssp. ofriconus n. ssp. W. 1964.

Scheloribatus parvus van Pletzen ssp. conglobatus n. ssp.

g) Famille Ceratozetidae Jacot 1925.

Africacarus calcaratus $n$. gen. n. sp. Wallwork 1965.

Hypozetes translamellotus n. sp. Wallwork 1965.

Ceratozetes rostroserratus n. sp. Wallwork 1965.

h) Famille Noct.lozetidoe Grandj. 1960.

Unguizetes reticuiatus n. sp. Wallwork 1965.

1) Famille Galumnidae Grand. 1936.

Golumna baloghi n. sp. Wallwork 1965.

Allogalumna pellucida n. sp. Wallwork 1965.

Trichogalumno microseto n. sp. Wallwork 1965.

Trichogalumna lunai Balogh.

j) Famille Phthirocaridae Perty 1841.

Hoplophorella africano n. sp. Wallwork 1965.

k) Famille Scutoverticidae.

Scutovertex fossotus n. sp. Wallwork 1965.

1) Famille Tectocepherdae.

Tecfocepheus vicarius Balogh.

\section{$4^{\circ}$ Variation des populations d'Oribates au cours d'une année.}

Le calcul des moyennes mensuelles de tous les Oribates récoltés pendant ces dernières années dans les douze gisements de Farcha, 
conduit au diagramme no 1 qui indique les variations des récoltes au cours d'une année.

On constate ainsi que dans les conditions de récoltes définies plus haut, le taux maxımum d'Oribates se situe en février, c'est-à-dire au cœur de la saison fraîche. Bien qu'assez élevé en mars et avril, le taux décroît et passe par un minımum à l'époque la plus chaude, en mai. Un deuxième maxımum, inférieur au premier, se rencontre au début de la saison des pluies. La période chaude et humide d'actobre -novembre ne semble pas favorable aux récoltes abondantes.

\section{$5^{\circ}$ Abondance des espèces.}

L'examen des différentes espèces récoltées dans tous les gisements et le calcul de leurfréquence montre que huit espèces d'Oribates se rencontrent régulıèrement, mais dans des pro- portions différentes; les autres n'étant toujours que faiblement représentées. Ces huit espèces classées par famille sont: Scheloribates perforatus, S. parvus conglobatus, S. fimbriatus ( $F$. Scheloribatidae); Africacarus calcaratus (F. Ceratozetidae) ; Galumna baloghi, Allogalumna pellucida (F. Galumnidae)* ; Oppia pilosella (F. Oppiidae) ; Unguizetes reticulatus ( $F$. Mochlozetidae).

Le diagramme $n^{\circ} 2$, établı d'après les valeurs moyennes montre la prédominance des deux espèces $S$. perforatus et $S$. fimbriatus; la première étant toujours très abondante quel que soit le lieu de récolte.

(*) La distinction entre les deux espèces citées n'ayant pas été effectuée régulièrement avec toute la rigueur nécessaire, leur importance relative est impossıble à préciser.

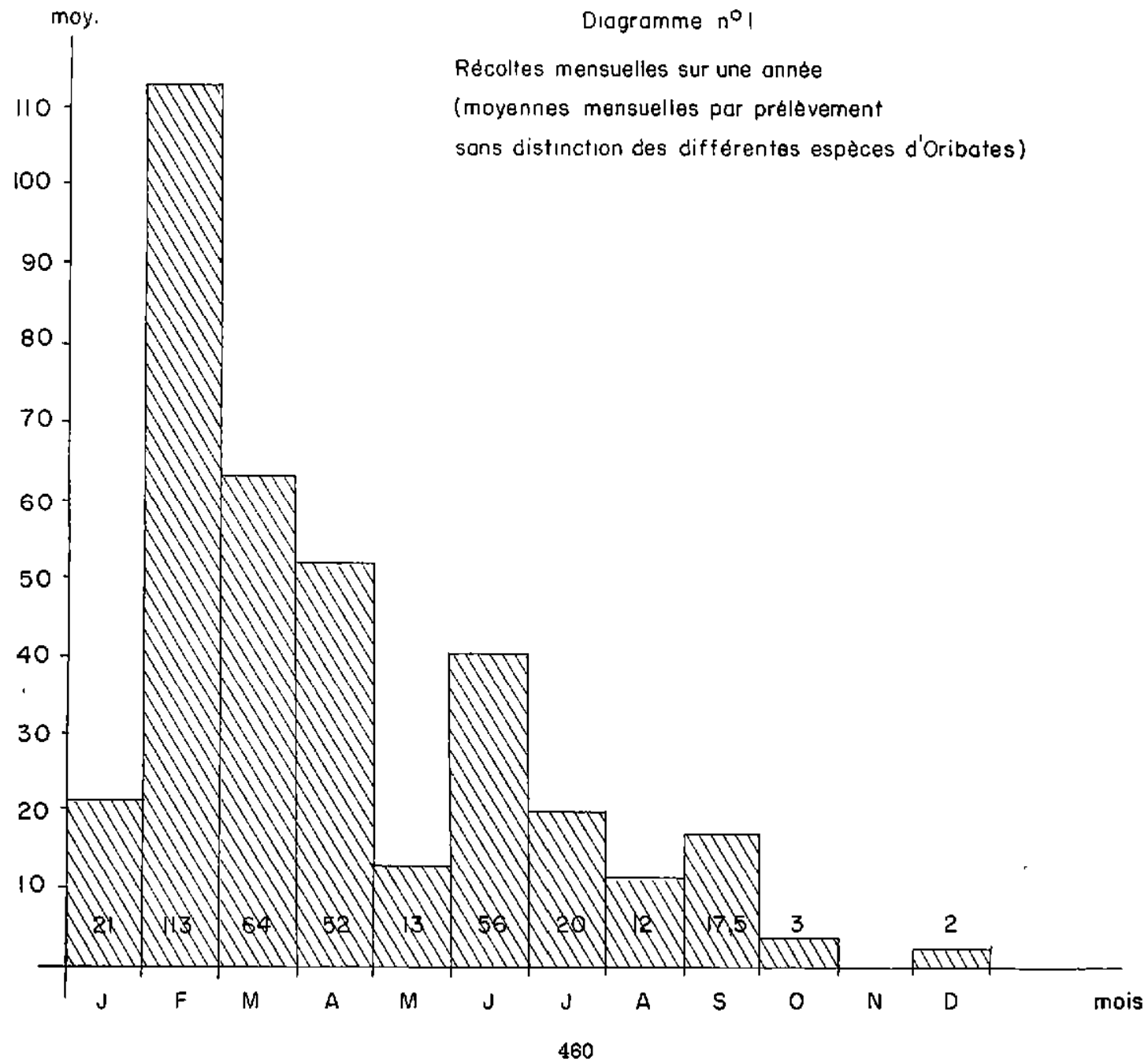




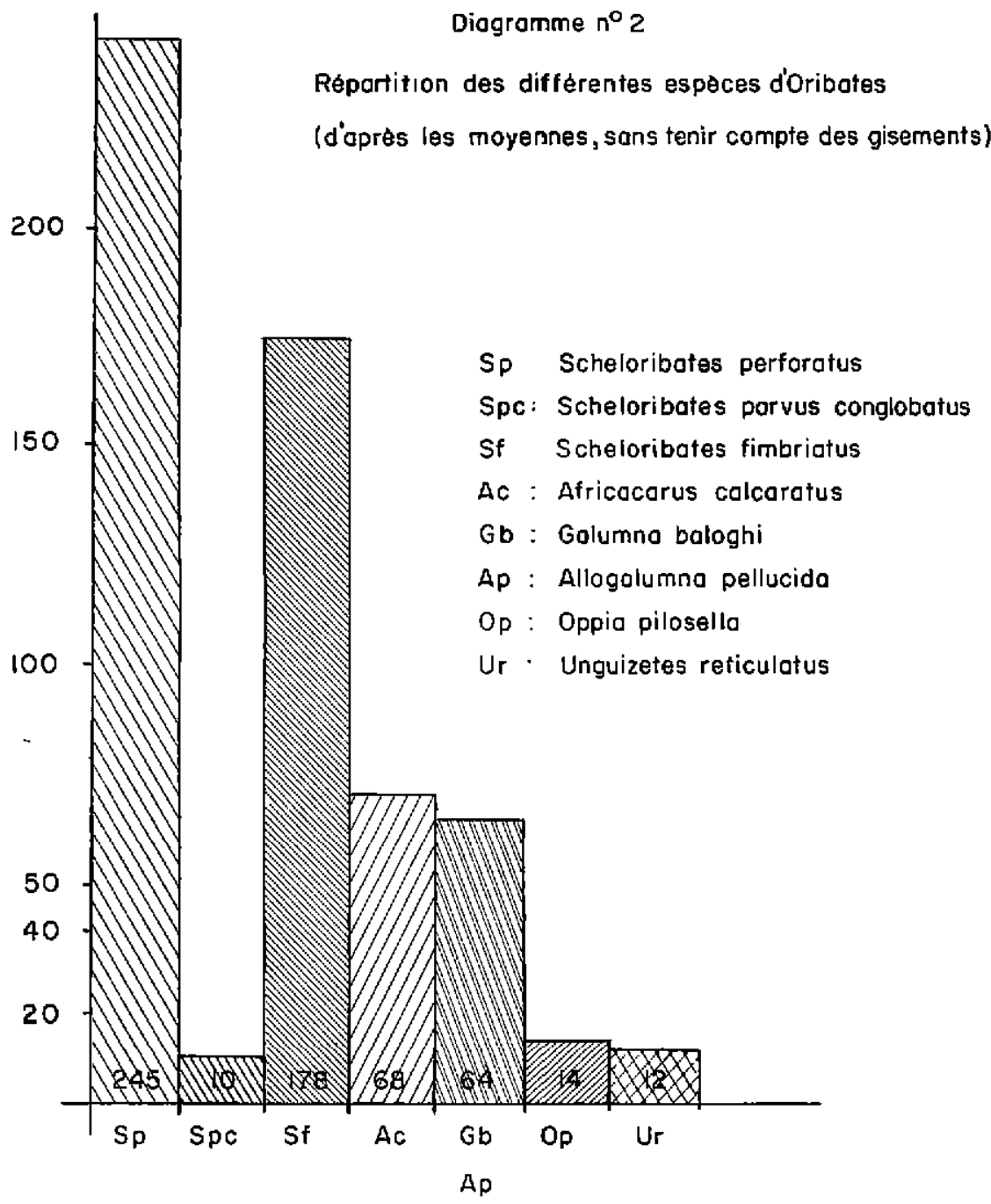

60 Classes d'abondance des espèces.

L'abondance relative des différentes espèces permet de les grouper selon six closses :

a) espèce très abondante, constamment rencontrée :

Scheloribotes perforotus ;

b) espèce abondante, mais limitée à certains gisements :

Scheloribates fimbriotus :

c) espèces fréquentes, assez régulièrement réparties :

Africacorus calcarotus,

Golumno baloghi,

Allogalumna pellucida ; d) espèces peu fréquentes :

Scheloribates parvus conglobatus,

Oppia pilosella,

Unguizetes reticulatus :

e) espèces rares :

Oppia heterosa,

Oppia fusiformis et sa forme lyroseta,

Hypozetes translamellatus,

Ceratozefes rostroserrofus,

Trichogalumna microseto,

Trichogalumno lunai,

Zygoribatula setosa :

f) espèces exceptionnelles :

Epilohmanno cylindrica,

Heptocarus hirsutus. 


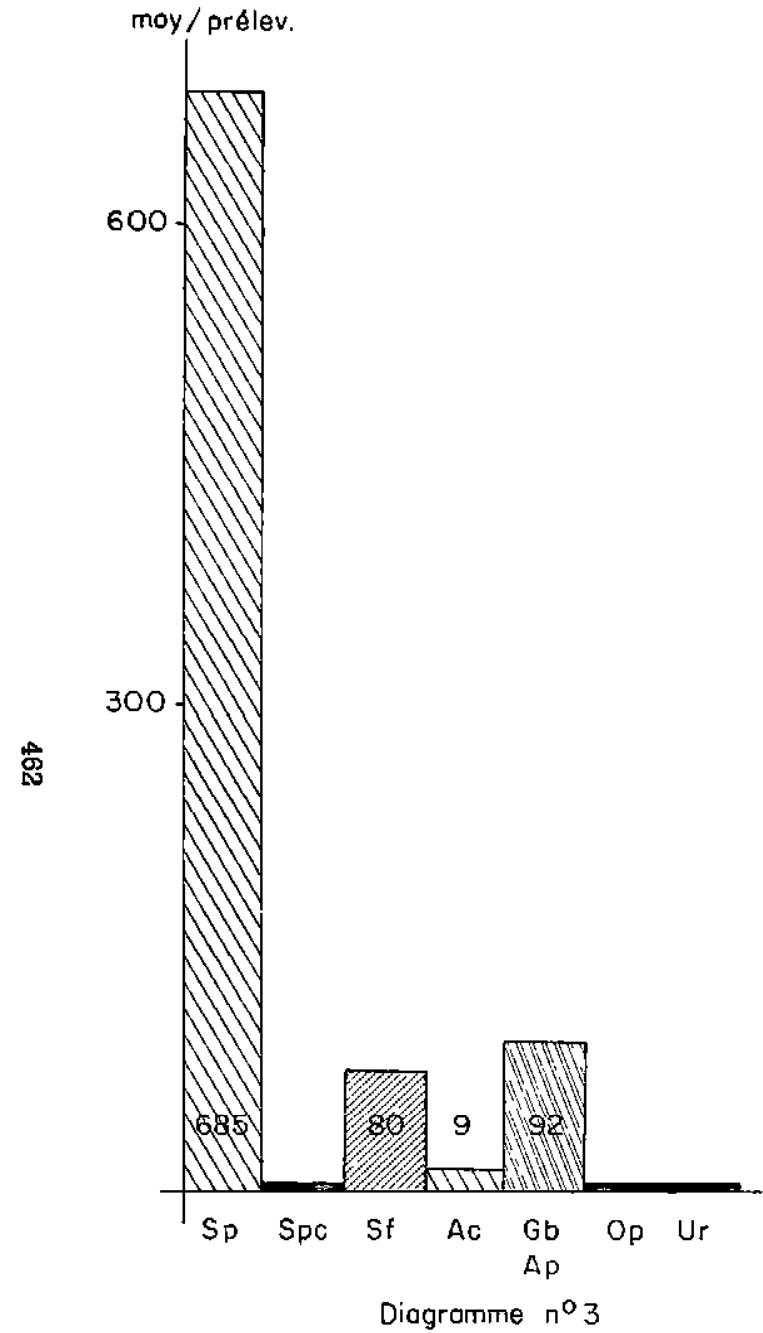

Gisements ombrogés

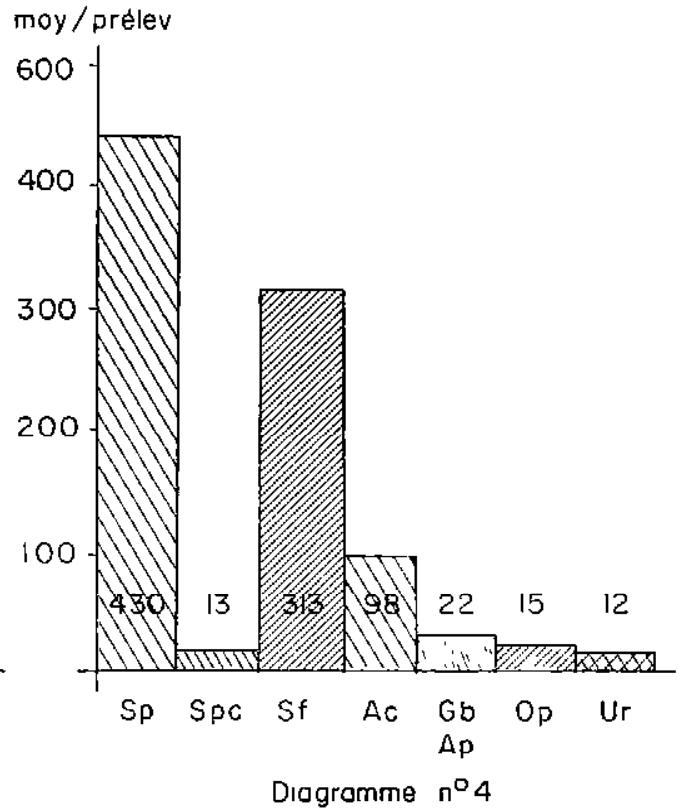

Gisements peu ombragés

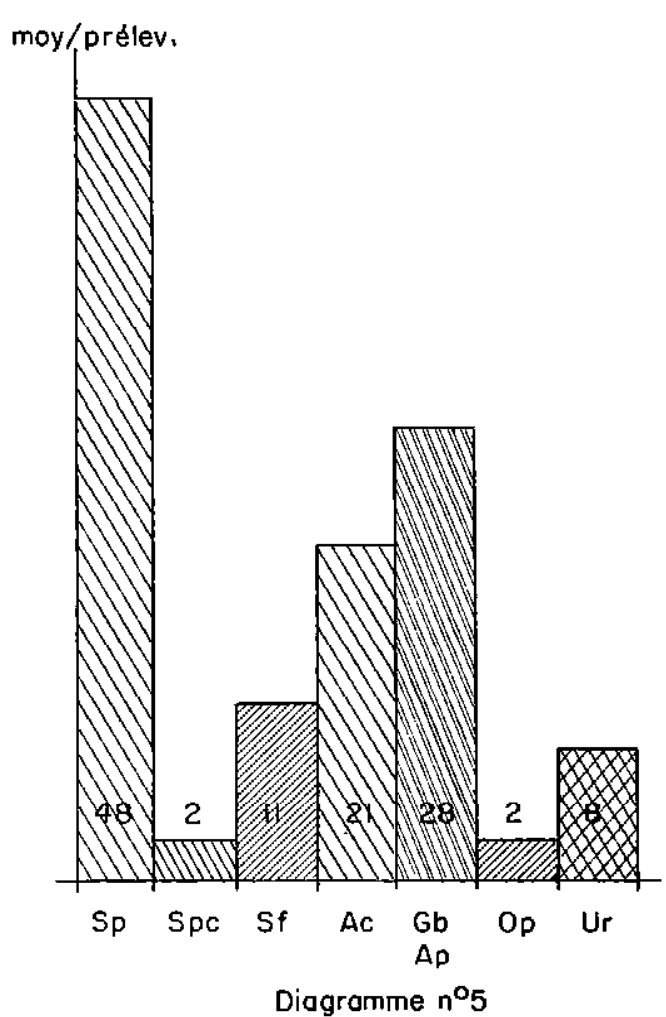

Gisements ensoleillés 
Passalozetes pectinatus,

Oribatula acuminato,

Hoplophorello ofricana,

Scutoverfex fossotus,

Tectocepheus vicorius.

70 Variation de la répartition des espèces selon le gisement.

Trols types de gisements peuvent être distingués selon l'insolation qu'ils recoivent : très ombragés, moyennement ombragés et presque continuellement ensoleillés. Le tablecu 1 et les diagrammes nos $3,4,5$ montrent les proportions dans lesquelles se rencontrent les principales espèces.

II ne semble pas que l'insolation ait une influence prépondérante sur la distribution des espèces qui existent en tout lieu, mais à des taux différents. Scheloribates perforatus, Galumna baloghi ef Allogalumno pellucida apparaissent bien plus abondants dans les terrains ombragés qu'ensoleillés. La plupart des autres espèces préfèrent ceux moyennement ombragés. Par contre, Oppia pilosella et Unguizetes reticulatus semblent fuir l'ombre, ils sont toujours fréquents dans les endroits à plus forte insolation.

TABLEAU $\mathrm{N}^{\circ} \mathrm{I}$

Principales espèces d'Oribates

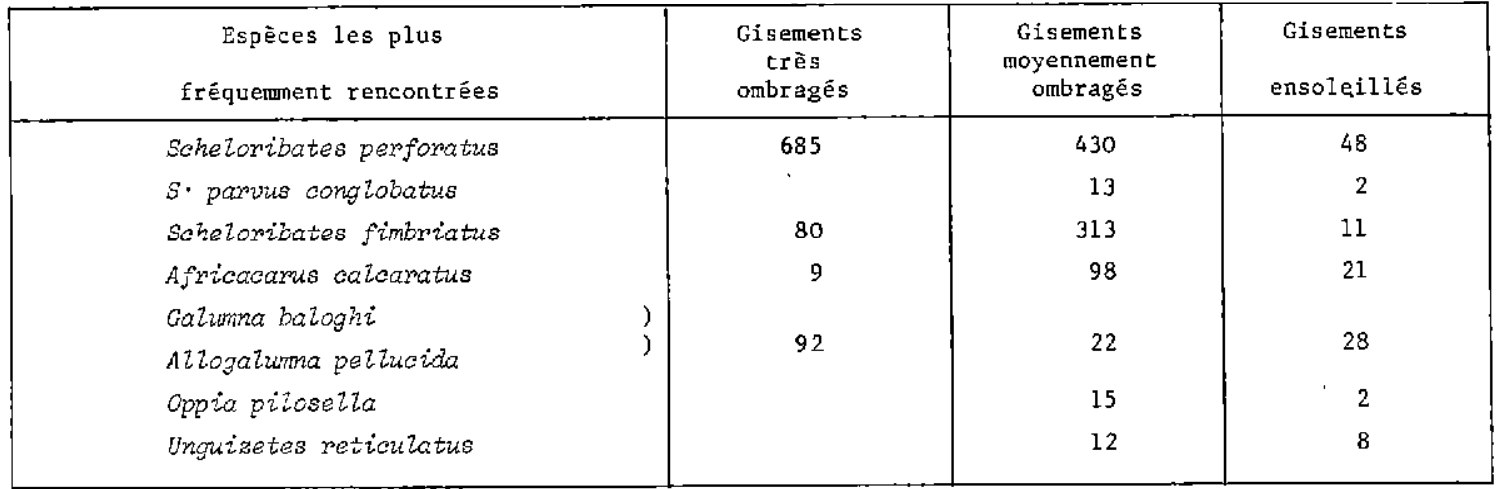

\section{ÉTUDE PARTICULIÈRE D'UN GISEMENT}

\section{A) Considérations générales.}

L'abondance constante des Oribates dans les différents prélèvements effectués dans l'un des gisements prospectés ( $n^{\circ}$ 11) l'ont fait retenir pour une étude plus précise.

La végétation y est caractérisée par deux Acacia sieberiana dont les ramecux enchevêtrés ne constituent qu'un seul ombrage, peu dense, sous lequel on trouve un autre Acocia ef un Balanifes aegyptiaco sous forme arbustive. La flore herbacée se compose de : Cassia tora $L$. (Papilionaceae), Achyranthes aspera L. (Amaranthaceae), Euphorbia hirto L. (Euphorbiaceae), ipomoea cortica (L.) Roth ex Roem. et Schult (Convolvulaceae), Setaria pollidefusca (Schum.) Stapf et Hubb (Graminaceae), Chloris prieurii Kunth (Graminacede), Brachioria ramoso (L.)
Stapf (Graminaceae), Digitoria sp. et Panicum sp. (Graminacede).

A l'époque où ont été effectués les prélèvements aux différents niveaux, ces herbes étaient en début de dessiccation.

Le diagramme $n^{\circ} 6$ indique la variation du taux mensuel d'Oribates récoltés dans ce gisement au cours d'une année. Dans l'ensemble les quantités d'Acariens prélevés chaque mois suivent les variations déjà observées pour l'ensemble des lieux de récoltes (voir diagramme no 1).

Les fréquences des espèces récoltées pendant la même période sont données par le diagramme no 7. Cinq espèces dominent, qui sont classées selon leur abondance : Scheloribates perforatus, Africacarus colcaratus, Scheloribates fimbriatus, Unguizetes reticulatus et Scheloribates parvus conglobatus. Non figurés sur le diagramme en 
Diagromme $n^{\circ} 6$

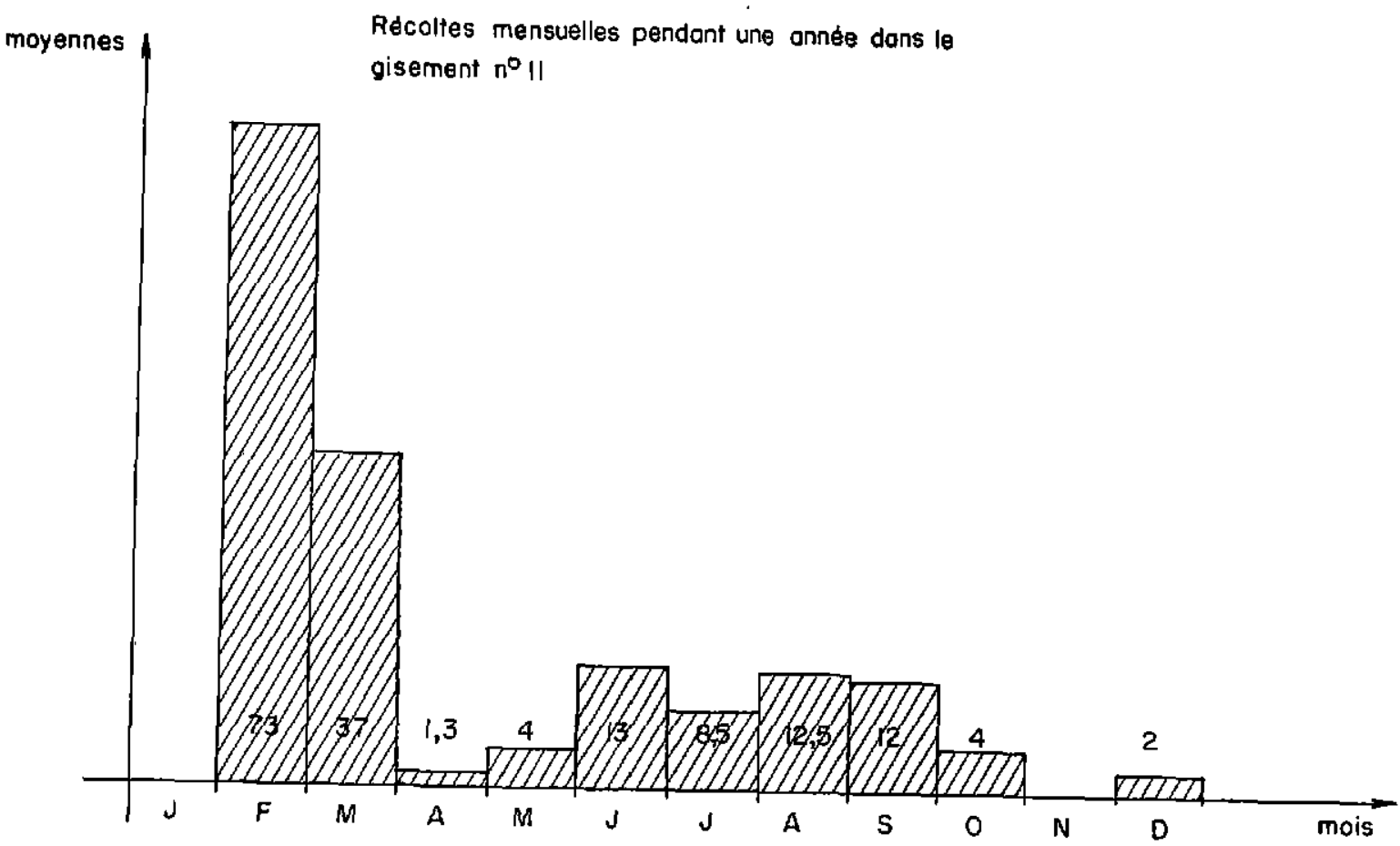

pour les abréviations, voir le diagromme $n^{\circ} 2$

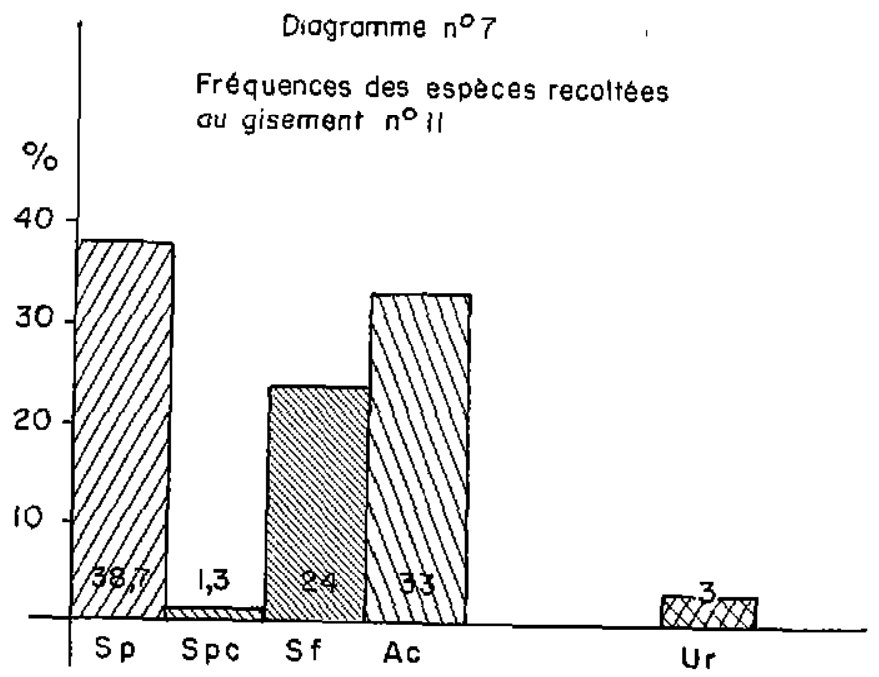

pour les abreviations, voir le diagramme $n^{\mathrm{O}} 2$ 


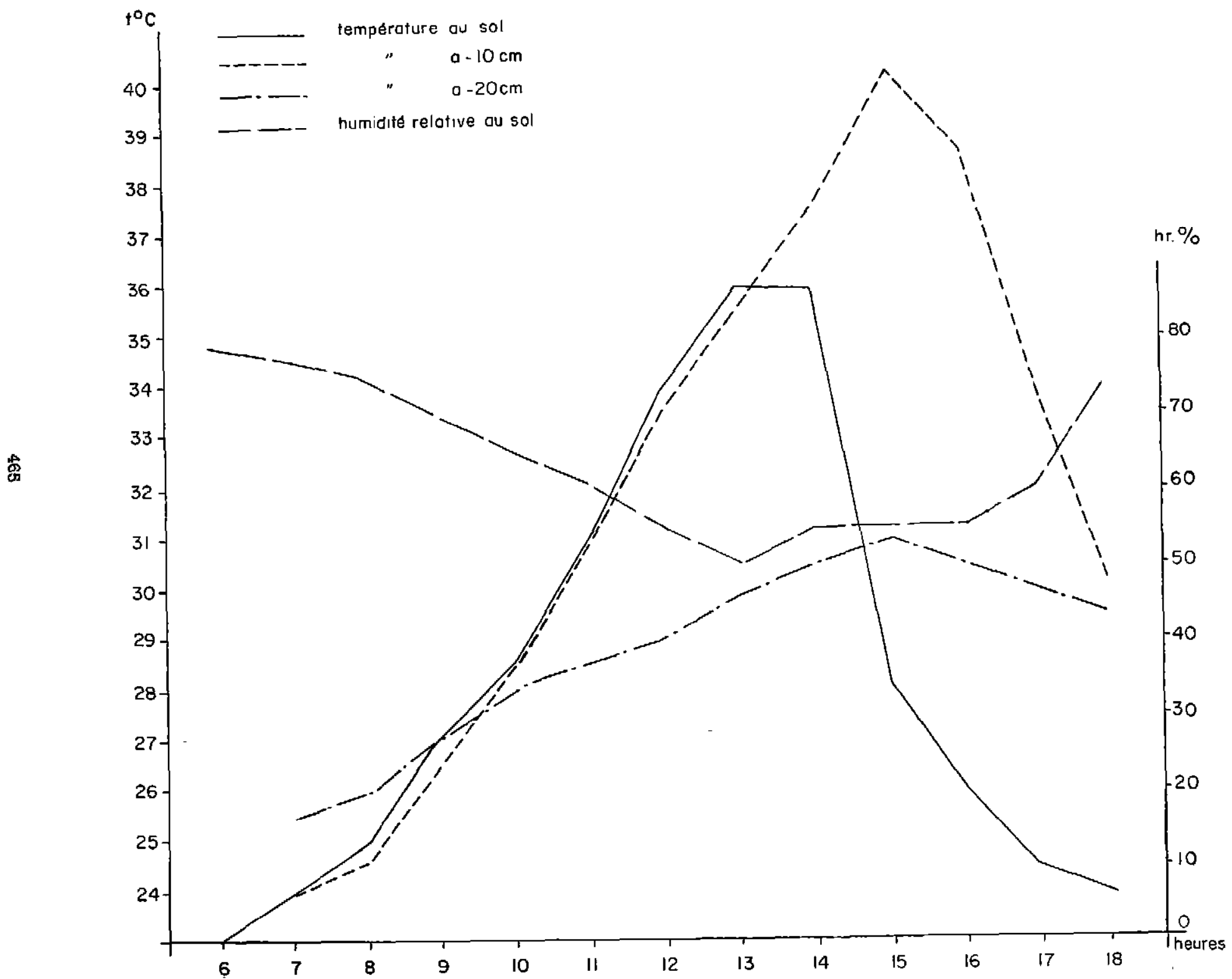


raison de leur trop faible densité, Galumna baloghs et Allogalumna pellucida sont cependant assez souvent rencontrés.

Scheloribates fimbriatus n'apparut très abondant qu'aux mais de février et mars et très peu fréquent le reste de l'année. L'étude de sa mıgration verticale ne sera donc pas envisagée puisque cette espèce n'a pas été rencontrée à l'époque choisie pour les observations décrites dans ce chapitre.

\section{B) Ełude détaillée du gisement no 11 .}

10 Récolte et nature des prélèvements.

L'étude particulière de ce gisement a été effectuée au cours des mois de septembre et octobre 1966 et repose sur des prélèvements effectués toutes les heures, de 6 heures à 18 heures, à trols niveaux différents: sur les herbes, à la surface du sol et à une profondeur de $15 \mathrm{~cm}$ environ.

$2^{\circ}$ Conditions écologiques prıncipales et répartition des populations d'Oribates.

Au cours de celte étude les températures on été mesurées à la surface du sol et aux profondeurs de 10 et $20 \mathrm{~cm}$; l'humidité relatıve a également été relevée d̀ la surface. Le graphique no 2 met en évidence les variations de ces éléments du microclimat au cours de la journée.

Le tableau $n^{\circ} 2$ rassemble les températures et humidités relevées à l'occasion des prélèvements.

On peut remarquer que les écarts entre les températures au sol et à $-10 \mathrm{~cm}$ sont fables jusqu'à 13 heures; l'écart s'agrandit ensuite et se maintient important jusqu'au soir, le refroldissement du sol étant plus lent et ne débutant qu'après 15 heures.

A dix centımètres de profondeur, la température atteint son maximum à 15 heures ; elle est alors de $140 \mathrm{C}$ plus élevée que celle de 7 heures. C'est à ce niveau que les variations de températures sont les plus grandes.

Par contre, à vıngt centimètres de profondeur, l'écart entre les extrêmes est rédult à $5,5^{\circ} \mathrm{C}$; la température maximale à ce niveau étant de $31^{\circ}$ est atteinte également à 15 heures.

Les températures enregistrées d̀ $-30 \mathrm{et}-40 \mathrm{~cm}$ (non figurées sur le graphique) sont pratiquement les mêmes. Entre 7 heures et 15 heures on note une différence de $4^{\circ} \mathrm{C}$; la température maximale étant de $30^{\circ} \mathrm{C}$.

L'humidité relative n'a pu être correctement mesurée qu'à la surface du sol ; elle passe par un minimum de 50 p. 100 à 13 heures ef dépasse 70 p. 100 avant 9 heures et à partir de 18 heures.

Mis à part Scheloribotes fimbriatus, non étudié pour des rasons indiquées plus haut, les autres espèces récoltées se retrouvent d̀ tous les niveaux, mais à des taux différents. Dans la matınée, c'est surtout en surface que les populations sont les plus nombreuses; elles migrent en profondeur dans l'après-midi. Mals pour l'ensemble de la journée, les pourcentages calculés à partir des récoltes obtenues d̀ chaque prélèvement indiquent que les Oribates sont plus nombreux en surface (voir tableau no 3).

30 Répartition des espèces selon les niveaux.

La répartition des différentes espèces d'Oribates aux différents niveaux est donnée, en pourcentages, pour la matınée et l'après-midi, dans le tableau no 4.

Ceci montre que Scheloribates perforotus et Africacarus calcaratus ont une répartition assez homagène. Ces deux espèces sont toutefors toujours plus abondantes en surface, sauf l'aprèsmidi où $S$. perforatus se rencontre davantage en profondeur.

Unguizetes reticulatus, plus abondant sur les herbes, apparaît comme l'espèce dont les déplacements sont les plus amples. II est toujours moins répandu en surface qu'aux autres niveaux. Cette remarque a pu être également faite dans tous les qutres gisements qui contıennent cette espèce.

Les Galumnidés rencontrés n'apparaissent que le matin, plus particulièrement en profondeur. Leur faible taux en surface ef leur absence sur les herbes indiquent qu'il s'agit d'une espèce très grégaire.

40 Variations de la répartition des différentes espèces dans les trois niveaux au cours de la journée.

Les remarques précédentes conduisent à examiner les variations de la répartition de chacune des espèces du gisement.

a) Scheloribates perforatus. Le tableau no 5 indique selon les niveaux les pourcentages récoltés aux différentes heures de la journée.

Le graphique $n^{\circ} 3$, tracé d'après ces pourcentages donne une idée des migratıons verticales de $S$. perforatus au cours de la journée. 
TABLEAL: $:^{\circ} I I$

Températures et degrēs hygrométriques

\begin{tabular}{|c|c|c|c|c|c|c|c|c|c|c|c|c|c|}
\hline Heures & 6 & 7 & 8 & 9 & 10 & I. 1 & 12 & 13 & 14 & 15 & 16 & 17 & 18 \\
\hline $\begin{array}{c}\text { Température } \\
\text { au sol }\end{array}$ & 23 & 24 & 25 & 27 & 28,5 & 31 & 34 & 36 & 36 & 28 & 26 & 24,5 & 24 \\
\hline $\begin{array}{r}\text { Température } \\
\text { à }-10 \mathrm{~cm}\end{array}$ & - & 24 & 24,5 & 26,5 & 28,5 & 31 & 33,5 & 35,5 & 37,5 & 40 & 39 & 34 & 30 \\
\hline $\begin{array}{c}\text { Température } \\
\text { à }-20 \mathrm{~cm}\end{array}$ & - & 25,5 & 26 & 27 & 28 & 28,5 & 29 & 30 & 30,5 & 31 & 30,5 & 30 & 29,5 \\
\hline $\begin{array}{l}\text { Humidité au } \\
\text { sol en p. } 100\end{array}$ & 78 & 76,5 & 75 & 70 & 65 & 62 & 55 & 50 & 55 & 55 & 55 & 60 & 75 \\
\hline
\end{tabular}

$+=$ Températures au ${ }^{\circ} \mathrm{C}$.

TABLEAU $\mathrm{N}^{\circ}$ III

Rêpartition (en pourcentage) des populations d'oribates selon les moments de la journée.

\begin{tabular}{|c|c|c|c|}
\hline Moment de la journëe & Herbes & Surface & Profondeur \\
\hline Matinée & 1,7 & 68,2 & 30,1 \\
\hline Après-midi & 1,3 & 39,1 & 59,6 \\
\hline Ensemble du jour & 1,5 & 58,4 & 40,1 \\
\hline
\end{tabular}

TABLEAU $\mathbb{N}^{*}$ IV

Rëpartition(en pourcentage) de diverses espèces d'Oribates selon les niveauk

\begin{tabular}{|l|c|c|c|c|c|c|}
\hline \multirow{2}{*}{ E s p è c e s } & \multicolumn{3}{|c|}{ Matinée } & \multicolumn{3}{c|}{ Après-midi } \\
\cline { 2 - 7 } & Herbes & Surface & Profondeur & Herbes & Surface & Profondeur \\
\hline Scheioribates perforatus & 73,8 & 87,3 & 86,7 & 78,4 & 80 & 84,2 \\
\hline Africacarus caloaratus & 8,8 & 10,2 & 8,3 & 18,9 & 19,7 & 13,6 \\
\hline $\begin{array}{l}\text { Galuma baloghi } \\
\text { Allogaluma peliucida }\end{array}$ & - & 0,8 & 1,9 & - & - & - \\
\hline Unguizetes reticulatus & 17,4 & 1,7 & 3,1 & 2,7 & 0,3 & 1 \\
\hline
\end{tabular}

\section{TABLEAU $\mathrm{N}^{\circ} \mathrm{V}$}

Pourcentages de $S$, perforatus rëcoltês à diverses heures du jour

\begin{tabular}{|c|c|c|c|c|c|c|c|c|c|c|c|c|c|}
\hline Heures & 6 & 7 & 8 & 9 & 10 & 11 & 12 & 13 & 14 & 15 & 16 & 17 & 18 \\
\hline Herbes & 1,5 & 0,3 & 5,5 & 3,6 & 0,5 & 0,6 & 1,9 & 0,2 & 0,9 & 8,5 & 0 & 0 & 0 \\
\hline Surface & 61,6 & 67,4 & 40 & 76,3 & 81,9 & 76,4 & 55,1 & 4,4 & 49,6 & 30,2 & 18,4 & 24 & 6,8 \\
\hline Profondeur & 36,9 & 32,3 & 54,5 & 20,1 & 17,6 & 24 & 43 & 95,4 & 49,5 & 61,3 & 81,6 & 76 & 93,2 \\
\hline
\end{tabular}


Grophique $n^{\circ} 3$

scheloribates perforatus

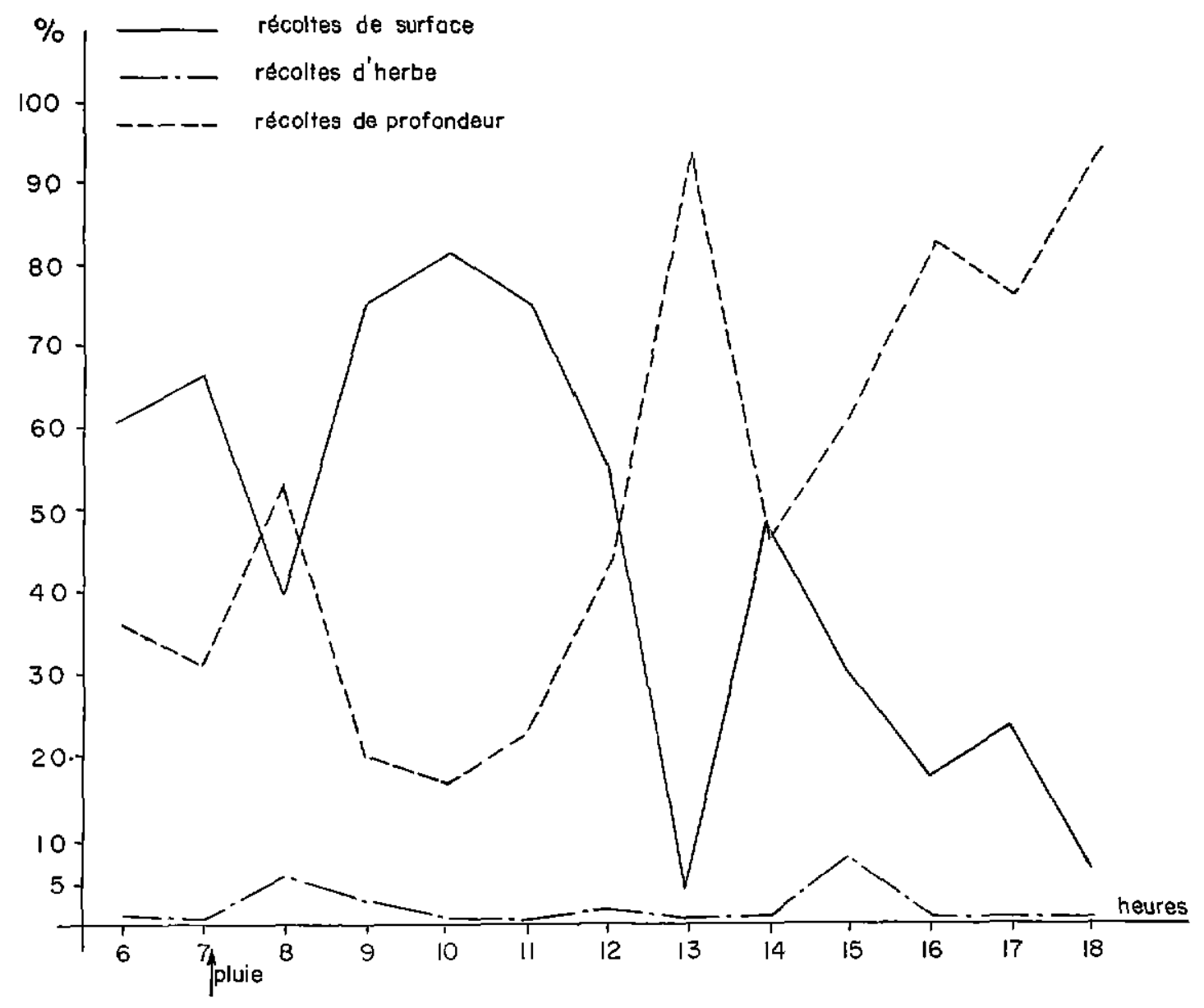

D'une manière générale, la population de surface augmente en même temps que la température durant la matinée. Mais à partir de 10 heures, ces Oribates entreprennent un déplacement vers la profondeur. Tout semble se passer comme si cette espèce voulait se maintenir d̀ un niveau où la température n'excède pas $28,5^{\circ} \mathrm{C}$ relevée à cette heure-là

La légère remontée observée à partir de 13 heures demeure encore inexpliquée. Par contre la diminution de la population de surface notée à 8 heures peut se justifier par l'intervention d'une petite pluie, peu après 7 heures; S. perforatus fuyant alors les zones mouillées de la surface pour se réfugier sur les herbes et en profondeur.

b) Unguizetes retıculatus. Le fableau $n^{0} 6$ et le graphique no 4 résument l'aspect des migrations verticales de cette espèce.

On remarque un parallélisme très net, du moins jusqu'à 14 heures, entre les variations des populations de surface et d'herbes. Cette dernière population est toujours nettement appréciable contrairement à ce que l'on observe pour les autres espèces. Ce fait indique pour $U$. rellculatus une grande mobilité et une tendance à rechercher la lumière et la chaleur. La petite pluie observée après 7 heures influe nettement sur la population présente sur les herbes et pratiquement pas sur celle voisine de la surface. Dans les deux cas, la chute de population observée à 11 heures reste inexpliquée; elle peut être due, soit à une faute de technique, soit à un phénomène biologique non encore décelé, soit 
TABLEAU $\mathrm{N}^{\circ} \mathrm{VI}$

Pourcentages d'U. reticulatus récoltés à diverses heures du jour

\begin{tabular}{|c|c|c|c|c|c|c|c|c|c|c|c|c|c|}
\hline Heures & 6 & 7 & 8 & 9 & 10 & 11 & 12 & 13 & 14 & 15 & 16 & 17 & 18 \\
\hline Herbes & 13,3 & 14,3 & 6,3 & 11,1 & 20 & 6,3 & 25 & - & - & - & - & - & - \\
\hline Surface & 50 & 42,8 & 43,7 & 57,7 & 60 & 43,7 & 75 & - & - & 40 & 11,2 & - & - \\
\hline Profondeur & 36,6 & 42,8 & 50 & 31,2 & 20 & 50 & - & 100 & - & 60 & 88,8 & 94,5 & 100 \\
\hline
\end{tabular}

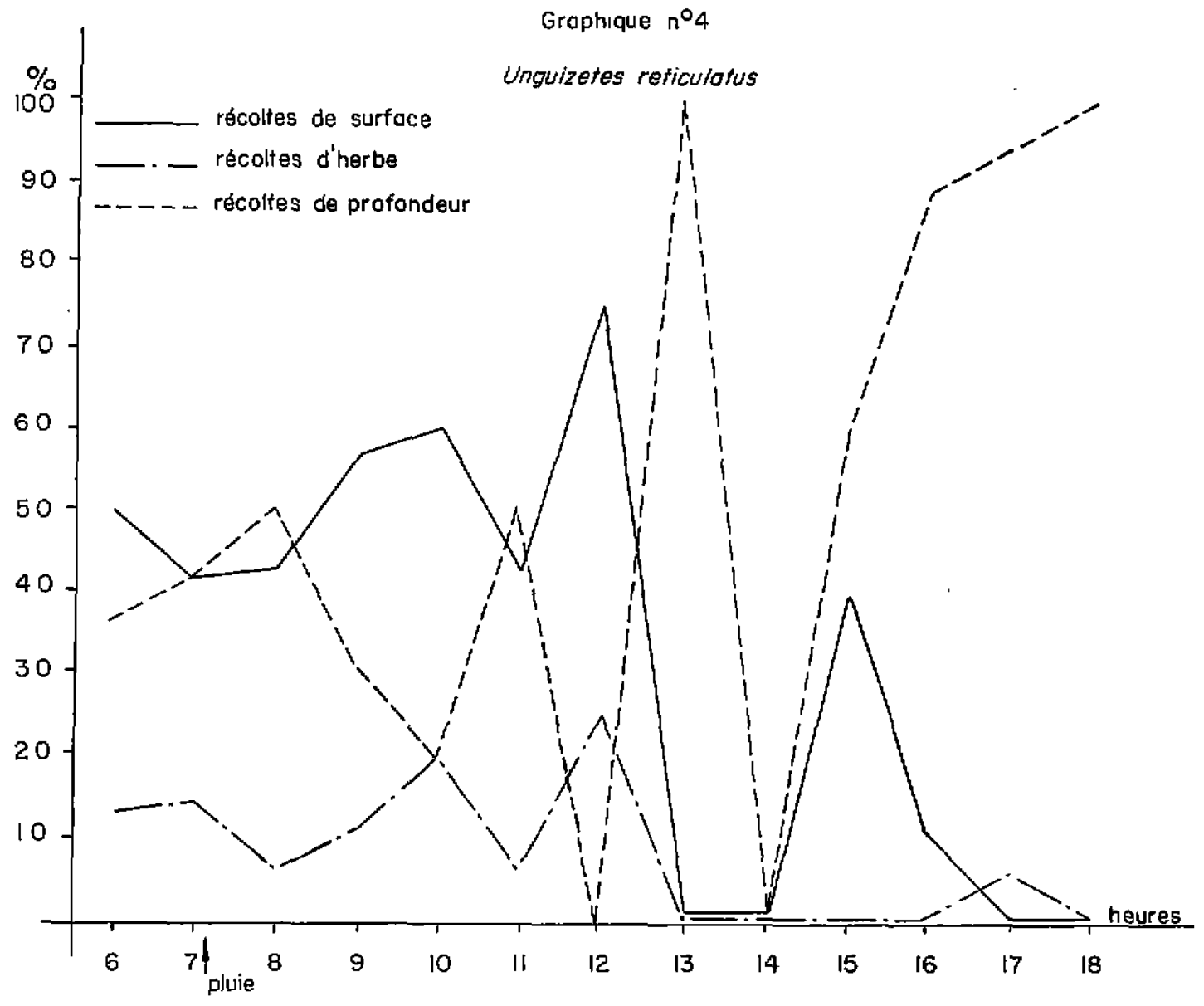

CAELEAU $N^{\complement}$ VII

Pourcentage d'A. calcaratus rếcoltês à diverses heures du jour.

\begin{tabular}{|c|c|c|c|c|c|c|c|c|c|c|c|c|c|}
\hline Heures & 6 & 7 & 8 & 9 & 10 & 11 & 12 & 13 & 14 & 15 & 16 & 17 & 18 \\
\hline Herbes & - & - & - & 28,5 & - & 2,8 & 3,6 & 0,6 & 3 & - & - & - & - \\
\hline Surface & 59 & 71 & 53,5 & 57,2 & 90,7 & 97,2 & 83,3 & 21,8 & 75 & 50 & 42 & 100 & 44 \\
\hline Profondeur & 41 & 29 & 46,5 & 14,3 & 19,3 & - & 13,1 & 77,6 & 22 & 50 & 59 & - & 56 \\
\hline
\end{tabular}




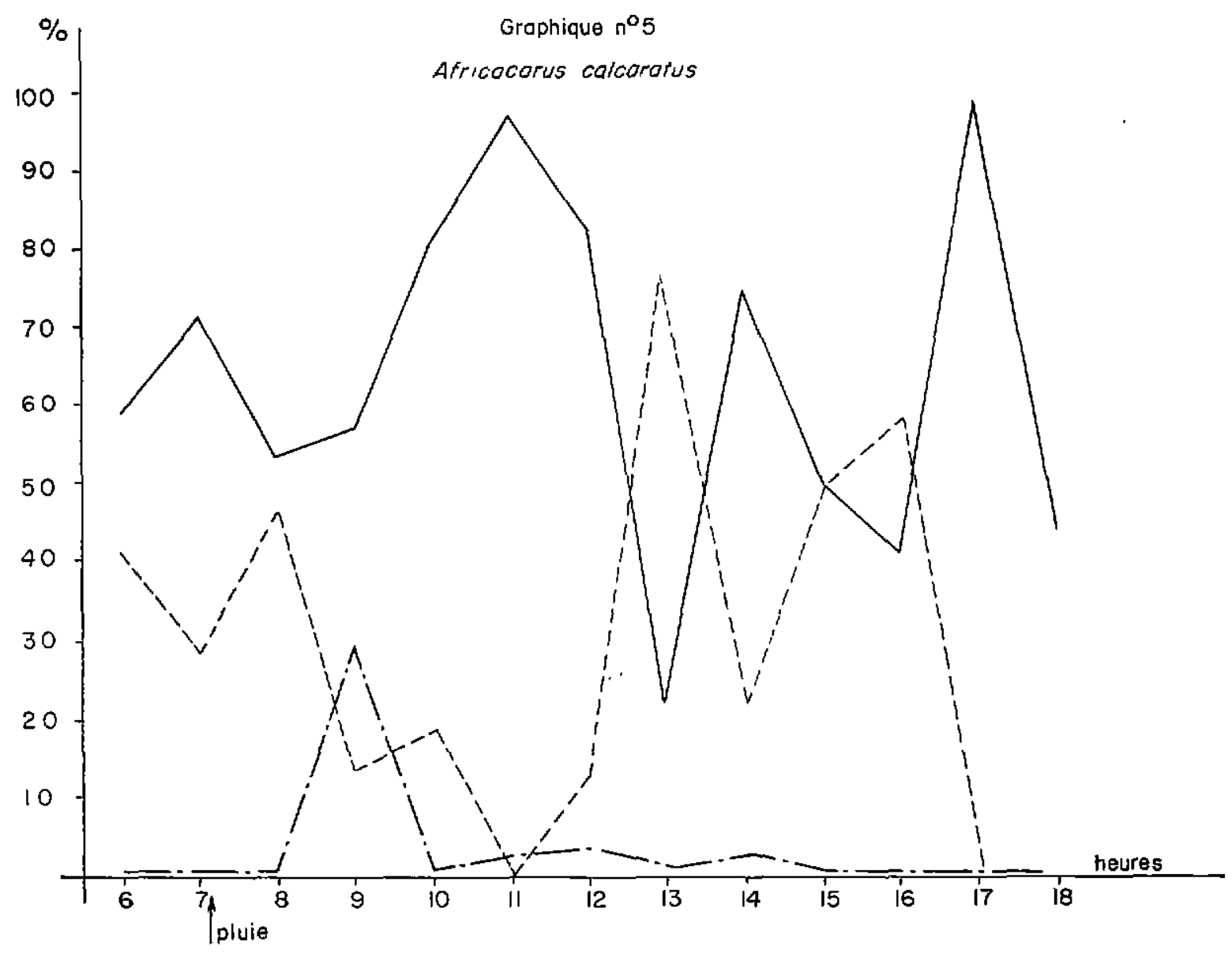

peut-être à un changement brutal dans l'insolation à l'endroit du prélèvement. A partir de 12 heures où la température de surface atteint 340 , les Unguizetes migrent en profondeur. La cause de la remontée observée vers 14 heures est là aussi inconnue.

\section{c) Africacarus calcaratus.}

L'examen du tableau no 7 et du graphique no 5 montre une augmentation constante de la population de surface au cours de la matinée, jusqu'd 11 heures où la température au sol atteint 310. A. calcaratus se réfugie alors en profondeur semblant rechercher une température inférieure à ce maximum.

La petite pluie du matin a également chassé ces Oribates de la surface. Là encore, la cause provoquant les migrations observées chez cette espèce au cours de l'après-midi reste inconnue.

d) Galumnidés : Golumno bologhi et Allogalumna pellucida (tableau no 8 et graphique $n^{\circ} 6$ ).
Aucun Galumnidé n'a été trouvé sur les herbes. Les deux espèces apparaissent très nettement comme des espèces de profondeur. Les taux relevés en surface sont toujours falbles. Dès 6 heures du matin, la population migre vers la profondeur et cette migration s'accélère après la pluie. Cependant dès 9 heures la population remonte en surface où elle se rencontre en totalité dès 10 heures. Mais aucune de ces espèces n'a été récoltée l'après-midi.

Dans l'ensemble, les chiffres relevés pour les Galumnidés sont toujours faibles et peu significatifs ; ils ne permettent guère de tirer des conclusions valables quant à leur biologie.

\section{CONCLUSION}

Les premiers résultats consignés dans cette note préliminaire apportent déjà quelques éclaircissements sur le comportement des Oribates dans la région sahélıenne. IIs indıquent 
TABLEAL ỸVIII

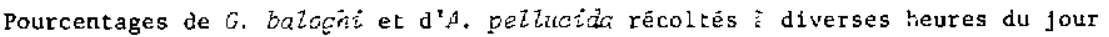

\begin{tabular}{|c|c|c|c|c|c|c|c|}
\hline Heures & 6 & 7 & 8 & 9 & 10 & 11 & 12 \\
\hline Heroes & - & - & - & - & - & - & - \\
\hline Surface & 100 & 81,8 & 27,2 & 17,6 & 100 & 100 & 100 \\
\hline Profordeur & - & 18,2 & 72,8 & 32,4 & - & - & - \\
\hline
\end{tabular}

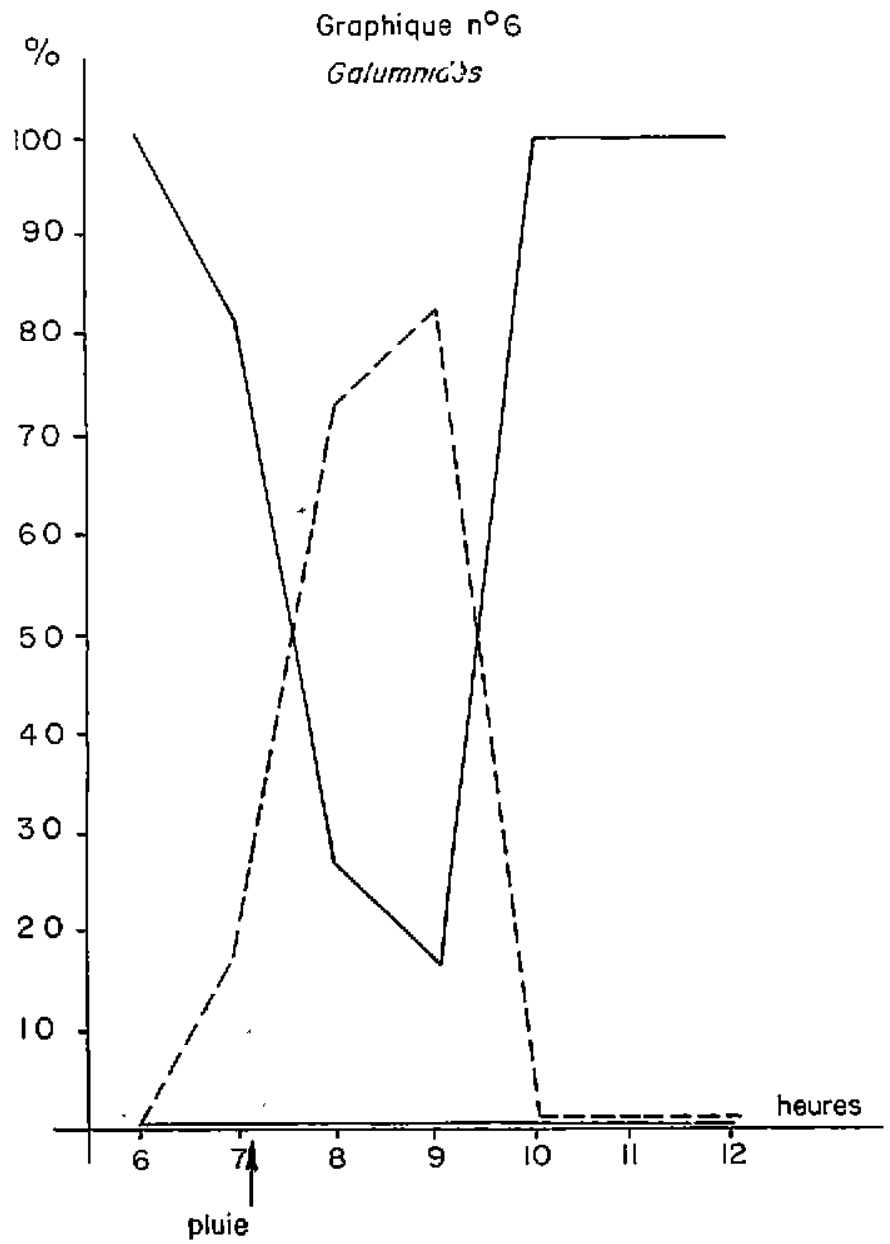

que sur les 23 espèces récoltées, 7 sont presque constamment trouvées et I'une d'elle, 5 . perforatus, particulièrement abondante en tout lieu.

Ces résultałs permettent en outre de préciser quelques affinités biologiques des espèces les plus fréquentes. S. perforatus ne semble pas supporter les températures supérieures à 290, mais se plait dans les couches superficielles du sol plus aérées. Unguizetes reticulatus apparaît comme une espèce plus mobile, préférant les couches supérieures et même la vie à l'air libre; elle ne redoute pas une insolation élevée. Africocarus calcarotus a une biologie apparemment voisine de celle de $S$. perforatus, mais supporte une température pouvant aller jusqu'à 310. Par contre, les Galumnidés s'opposent très nettement à ces trois espèces. En général toujours moins nombreux, les représentants de cefte famille ne se rencontrent jamais sur les herbes et rarement en surface. Ils ne se plaisent 
qu'en profondeur, fuyant ainsi l'insolation et la lumière. Ces résultats sont en accord avec quelques remarques effectuées à l'occasion d'élevages en Laboratoire.

Ces premières remarques permettront donc d'orienter avec plus de précisions les prochaines recherches sur la biologie des Oribates au Tchad.

Les difficultés d'interpréter actuellement certains mouvements de population au cours de la journée, notamment dans l'après-midi, doivent inciter à préciser l'étude de l'humidıté, de son action conjointe avec la température ainsı que tout autre facteur écologique secondaire non encore mis en évidence.

Les variations d'humidité parassent, encore plus que celles des températures, avorr une influence déterminante sur le comportement des Oribates.

Si l'on considère les Oribates en tant qu'hôtes intermédıaıres du Cestode Stilesia globıpunctata, les remarques précédentes présentent l'intérêt de confirmer les conclusions faites dans une publication antérieure. Les expériences de transmission et la recherche des cysticercoïdes dans les
Oribates avaient permis de reconnaître que dans les conditions naturelles, les meilleurs vecteurs étaient d'abord les Galumnidae (avec 19,3 p. 100 d'infestés), puis Africacarus calcaratus (4,2 p. 100) et enfin Scheloribates perforatus (avec 2,93 p. 100). Ces résultats sont apparemment contradictoires avec les remarques sur la biologie de ces espèces. En effet, le meilleur vecteur (Golumnidae) est peu répandu et pratiquement hors de portée du mouton, alors que $S$. perforotus, moins bon vecteur est par contre très répandu ef facilement accessible aux ovins. S. perforatus compense son faible pouvorr transmetteur par son extrême abondance et par sa grande dispersion à la surface du sol et sur les herbes. Là encore, les vecteurs les plus nombreux, bien que moins adaptés, prennent le pas sur les plus qualifiés lorsque ceux-ci sont les moins nombreux.

\section{Institut d'Elevage et de Médecine vétérinaire des Pays Tropicaux. Laboratorre de Farcha-Fort-Lomy, (TChad).}

\title{
SUMMARY
}

\section{Preliminary note on the Ecology of some Oribatideae species in Chad}

\begin{abstract}
Many collections of Oribatideas acarian have been made in Chad since 1963 in order to sludy the transmission of the cestod Stilesia globipunctata of sheep. Observations on their ecology have been recorded.

Twenty three Oribatideae species have been collected in various locations of the Farcha's laboratory estate, which is situated in the sahel ian area. The climatic conditions of this area briefly reminded.

Oribates have been collected in great quantity all over the year, but they are particularly numerous during the cool season.

Amongst the species found, a few only are always found, whatever the season and the place are; all the other are rare and even exceplionnal.

After the rainy season systematic survey has been carried out in a place where the Oribates were particularly numerous. Samples have been taken at each hour of the day and at three different levels, Interpretation of the results showed some particulars of the biology of the most frequent species.

Close relationship between density and movements of the Oribatidease populations and importance of infestation of sheep has been evidenced.
\end{abstract}

\section{RESUMEN}

Primeras notas ecológicas concernientes a algunas especies de oribatos en Chad

Las numerosas recogidas de Acaridos Oribatos efectuadas en Chad desde 1963 para estudiar la transmisión del céstodo de la oveja Stilesia globipunctata permitieron la observación de la ecalogia de ellos. Se recogieron veinte y tres 
especies de Oribatos en varios sitios de los terrenos del Laboratorio de Farcha. situado en zona saheliana. cuyas características climáticas y botánicas estan notadas brevemente. Siempre se encueniran los oribatos con abundancia a lo largo del año, pero son particularmente numerosos durante la estación fresca.

Entre las especies empadronadas, solo algunas se encuentran cualesquiera que sean la estación y el sitio ; todas las oiras son muy pocas, aún escasas.

Despues de la estación de las lluvias, se hicıeron estudios sistemáticos en una zona particularmente rica. Se efectuaron tomas en cada hora del día y en tres niveles diferentes La interpretación de los resultados muestra algunos aspectos del comportamiento de las especies más abundantes. Asi se demostró una relación entre las densidades y las mudanzas de las populaciones de Oribatos y las intensidades de las infestaciones de las ovejas.

\section{BIBLIOGRAPHIE}

AUBREVILLE (A.). - Climats, forêts et désertification de l'Afrique tropicale. Soc. Ed. géog. mor. col. Paris. 1949.

GRABER (M.) et GRUVEL (J.), - Transmission au mouton de Stilesia globipunctata Rivolta 1874 (Cestoda, Anoplocephalidae) à partir de divers Acariens Oribates. C. R. Acod. Sci. Poris, 1964, †. 259, p. 2680-2682.

GRABER (M.) et GRUVEL (J.). - Note préliminaire concernant la transmission de Stilesia globipunctata Rivolta 1874 du mouton par divers Acariens Oribates. Rev. Elev. Méd. vét. Poys trop. 1964, 17, 3 (467-76).

GRUVEL (J.) et GRABER (M.). - Observations sur quelques stades d'évolution d'Oribates récoltés au Tchad. Rev. Elev. Méd. vét. Pays trop. 1964, 17, 3, (571-573).

GRUVEL (J.) et GRABER ( $M_{1}$ ). - Récolite ef mise en élevage d'Acariens Oribates dans les conditions tchadiennes. Rev. Elev. Méd. vét. Pays trop. 1964, 17, 3 (575-580).

GRABER (M.) et GRUVEL (J.). - Les vecteurs de Stilesia globipunctata Rivolta 1874, du mouton. Rev. Elev. Méd. vét. Pays trop. 1967, $20,2,261-71$.

WALLWORK (J. A.). - Some Oribatei (Acari : Cryptostigmata) from Tchad Ist. series Rev. Zool. et Bot. africoines ; 1964, LXX, fasc. 3-4, 353-385.

WALLWORK ( $($. A.). - Some Oribatei (Acari : Cryptostigmata) from Tchad. 2d. series. Rev. Zool. et Bot. ofricoines; 1965, LXXII, fasc. 1-2, p. 83-108.

WALLWORK (J. A.), -- Some Oribatei (Acari : Cryptostigmata) from Tchad. 3d. series. ... sous presse. 